\title{
Determination of the carrier-envelope phase of few-cycle laser pulses with terahertz-emission spectroscopy
}

\section{MARKUS KREß ${ }^{1 *}$, TORSTEN LÖFFLER ${ }^{1}$, MARK D. THOMSON ${ }^{1}$, REINHARD DÖRNER ${ }^{2}$, HARTMUT GIMPEL $^{3}$, KARL ZROST ${ }^{3}$, THORSTEN ERGLER ${ }^{3}$, ROBERT MOSHAMMER ${ }^{3}$, UWE MORGNER ${ }^{3,4}$, JOACHIM ULLRICH $^{3}$ AND HARTMUT G. ROSKOS ${ }^{1}$}

\author{
${ }^{1}$ Physikalisches Institut, Johann Wolfgang Goethe-Universität, Max-von-Laue-Str. 1, D-60438 Frankfurt (M), Germany \\ ${ }^{2}$ Institut für Kernphysik, Johann Wolfgang Goethe-Universität, Max-von-Laue-Str. 1, D-60438 Frankfurt (M), Germany \\ ${ }^{3}$ Max-Planck-Institut für Kernphysik, Saupfercheckweg 1, D-69117 Heidelberg, Germany \\ ${ }^{4}$ Institut für Quantenoptik, D-30167 Hannover, Germany \\ *e-mail: m.kress@physik.uni-frankfurt.de
}

$\mathbf{T}$ The availability of few-cycle optical pulses opens a window to physical phenomena occurring on the attosecond timescale. To take full advantage of such pulses, it is crucial to measure ${ }^{1-4}$ and stabilize ${ }^{1,2}$ their carrier-envelope (CE) phase, that is, the phase difference between the carrier wave and the envelope function. We introduce an approach to determine the CE phase by down-conversion of the laser light to the terahertz $(\mathrm{THz})$ frequency range by means of plasma generation in ambient air, an isotropic medium where optical rectification (downconversion) in the forward direction is only possible if the inversion symmetry is broken by electrical or optical means ${ }^{5-10}$. We show that few-cycle pulses directly produce a spatial charge asymmetry in the plasma. The asymmetry, associated with $\mathrm{THz}$ emission, depends on the $\mathrm{CE}$ phase, which allows determination of the phase by measurement of the amplitude and polarity of the THz pulse.

The ability to measure ${ }^{1-4}$ and stabilize $e^{1,2}$ the CE phase of fewcycle laser pulses, that is, the ability to completely control ultrashort high-intensity electromagnetic fields, provides access to a whole series of novel physical phenomena, and creates options for their coherent control. An example is the manipulation of the electron recombination process during 're-collision" ${ }^{\text {'1 }}$, with the aim of fully controlling the generation of both intense vacuum-ultraviolet higher-harmonic radiation ${ }^{12,13}$ as well as of high-intensity single attosecond pulses. The progress has led to the establishment of a new field of research, 'attosecond science', with highlights of recent work including the efficient tomography of molecular wavefunctions by coherent diffraction of rescattered electrons ${ }^{14}$, as well as the preparation of high-intensity, monochromatic femtosecond electron beams evolving from 'bubble generation ${ }^{15-18}$. Many of the phenomena studied depend critically on the CE phase. Examples include the dynamics of above-threshold ionization $(\mathrm{ATI})^{4}$, light-induced non-sequential double ionization ${ }^{19}$, Gouy

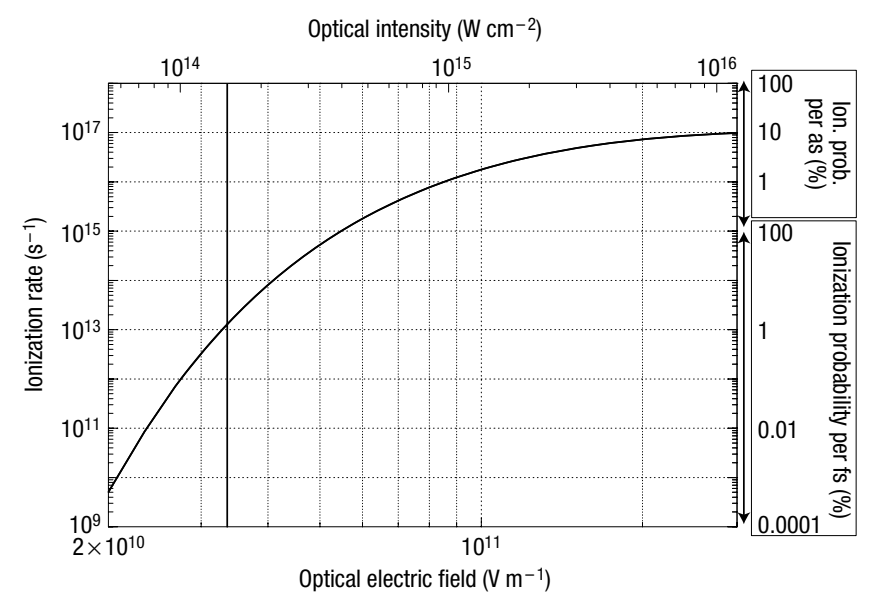

Figure 1 lonization rate for $\mathrm{N}_{2}$ versus electric field strength on the basis of the static-tunnelling theory as a function of the optical field strength, and the light intensity respectively. The calculation was carried out with the help of equation (1). An ionization rate of $10^{15} \mathrm{~s}^{-1}$ corresponds to a probability of $100 \%$ for a molecule to be ionized during a time interval of $1 \mathrm{fs}$. Note that the probability for ionization of a molecule has a value of $1 \%$ per fs at a threshold field of $3.3 \times 10^{10} \mathrm{~V} \mathrm{~m}^{-1}$ (vertical black line), and that the ionization probability per fs already becomes $100 \%$ at $5.5 \times 10^{10} \mathrm{~V} \mathrm{~m}^{-1}$, that is, at less than twice the threshold field.

phase observation ${ }^{20}$, and quantum interference in photocurrents ${ }^{21}$. An exciting prospect is the application of the CE phase as a coherent-control tool, for example, to steer bound electrons in molecules ${ }^{22}$. 


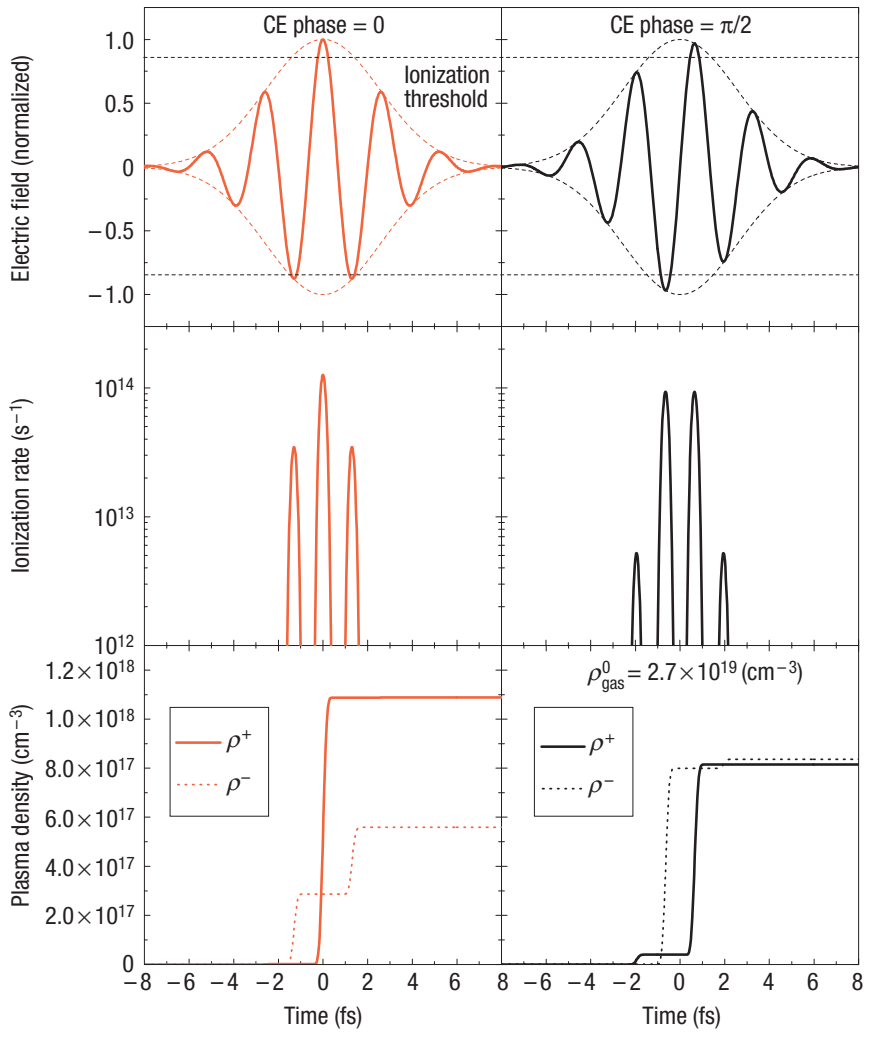

Figure 2 Optical electric field, ionization rate and plasma densities as a function of time for CE phases $\varphi_{\mathrm{CE}}=\mathbf{0}$ and $\varphi_{\mathrm{CE}}=\pi / 2$. The plasma densities $\rho^{+}$ and $\rho^{-}$, generated at positive and negative field polarities, respectively, are calculated with equations (2) and (3) of the Methods section. The ionization threshold is the arbitrarily chosen electric-field value where the ionization probability is $1 \%$ per fs.

Few-cycle pulses can be represented as $E(t)=$ $E_{0}(t) \cos \left(\omega t+\varphi_{\mathrm{CE}}\right)$, with $\omega$ being the angular frequency of the carrier wave and $E_{0}(t)$ being the envelope function with its maximum at $t=0$. Here $\varphi_{\mathrm{CE}}$ defines the $\mathrm{CE}$ (or absolute) phase. The state-of-the-art method for its determination in the case of amplified laser pulses is stereo $\operatorname{ATI}^{23,24}$, whereas the alternative f-to-2f interferometry method ${ }^{1,2}$, in practice, only tracks changes in the CE phase. In this letter, we present a new approach for CE phase determination on the basis of down-conversion into the $\mathrm{THz}$ frequency range in a laser-generated ambient air plasma. The concept relies on the detection of the $\mathrm{THz}$ electromagnetic pulses emitted during photo-ionization of the air by the focused few-cycle pulses. The method is easy to apply, because it does not require a vacuum chamber and relies on standard optoelectronic $\mathrm{THz}$ detection technology $y^{5,8}$.

THz-pulse emission from laser-generated plasmas has been investigated in the past, but only with much longer laser pulses. There, the transient electric currents responsible for the $\mathrm{THz}$ radiation have their origin either in ponderomotive forces ${ }^{25}$, the Coulomb force of an externally applied d.c. bias ${ }^{5,6}$, or an optical 'a.c. bias' achieved by superimposing the light pulses with their own second-harmonic radiation ${ }^{7-10}$. The THz-wave generation mechanism for the latter has been discussed in terms of a four-wave mixing process ${ }^{7,9,10}$. For the present case where the pulse spectra are octave-spanning, four-wave mixing results in a low-frequency $(\Omega \rightarrow 0)$ polarization: $P\left(\Omega=\omega_{1}+\omega_{2}-\omega_{3}\right) \propto$ $\sqrt{I\left(\omega_{1}\right) I\left(\omega_{2}\right) I\left(\omega_{3}\right)} \cos \left(\varphi_{\mathrm{CE}}\right)$, because the CE phases $\varphi_{\mathrm{CE}}$ of all

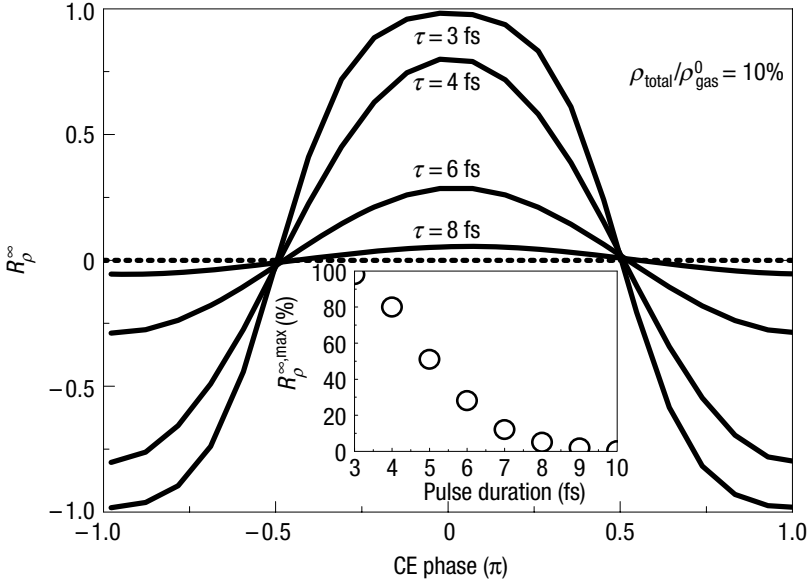

Figure 3 Asymmetry parameter $\boldsymbol{R}_{\rho}^{\infty}$ of the plasma density versus CE phase for several pulse durations. $R^{\infty}$ is proportional to the macroscopic quasi-d.c. dipole moment, which is responsible for the emission of the $\mathrm{THz}$ radiation. The pulse intensity is chosen so that a plasma density $\rho_{\text {total }}$ of $10 \%$ of the molecule density $\rho_{\text {gas }}^{0}$ is obtained. Inset: Maximal value of $R_{\rho}^{\infty}$ at $\varphi_{\mathrm{CE}}=0$ as a function of the pulse duration.

three input waves with spectral intensities $I\left(\omega_{i}\right)$ are the same (as all frequency components arise from the same near-transform-limited few-cycle pulse). Therefore, the CE phase is directly imparted onto the single-cycle $\mathrm{THz}$ pulse, and thus can be determined by means of electro-optic measurement of the $\mathrm{THz}$ field amplitude. The same argument concerning the transfer of the $\mathrm{CE}$ phase to the $\mathrm{THz}$ field can be readily generalized to the case of higher-order nonlinear contributions. Although such a wave-mixing process illustrates the mechanism for $\mathrm{CE}$ phase-sensitive $\mathrm{THz}$ generation, we will give an alternative description on the basis of static-tunnelling theor $y^{26}$, which explains the origin of nonlinear polarization on a microscopic level. For simplicity, we assume that the air only consists of $\mathrm{N}_{2}$. Figure 1 shows the calculated ionization rate $w_{\text {tun }}$ for $\mathrm{N}_{2}$ at a pressure of $1 \mathrm{~atm}$, and the ionization probability rate of a single molecule, respectively. The strongly nonlinear intensity dependence of the ionization rate translates into temporal ionization patterns as illustrated in Fig. 2. The upper panels display the two pulse shapes considered here, both with the same pulse duration of $\tau=6$ fs (full-width at half-maximum), the same centre wavelength of $\lambda=800 \mathrm{~nm}$, and the same peak electric field of the envelope of $E_{\mathrm{opt}}^{0}=4.2 \times 10^{10} \mathrm{~V} \mathrm{~m}^{-1}$, but with different CE phases, $\varphi_{\mathrm{CE}}=0$ and $\varphi_{\mathrm{CE}}=\pi / 2$. The middle panels of Fig. 2 show the time evolution of the ionization rates for the two cases. A common signature is that only the three or four most-intense half-cycles of the pulses ionize a significant amount of molecules. The ionization rate at each half cycle, however, depends sensitively on the $\mathrm{CE}$ phase. This becomes even clearer when we calculate the temporal evolution of the plasma density. We distinguish two quantities, $\rho^{+}(t)$ and $\rho^{-}(t)$, which describe the densities of free electrons produced at positive and negative polarity of the optical laser field, respectively. The total density of free electrons is given by $\rho_{\text {total }}=$ $\rho^{+}(t)+\rho^{-}(t)$. The lower pair of panels of Fig. 2 show that for $\varphi_{\mathrm{CE}}=0$, the total plasma density generated at negative field polarity is significantly smaller than the density for positive polarity. For $\varphi_{\mathrm{CE}}=\pi / 2$, the densities only differ a little after the pulse has passed through the focus.

In this simplified model, we assume that electrons generated at positive field polarity experience a displacement in the positive field direction due to acceleration in the laser field and 


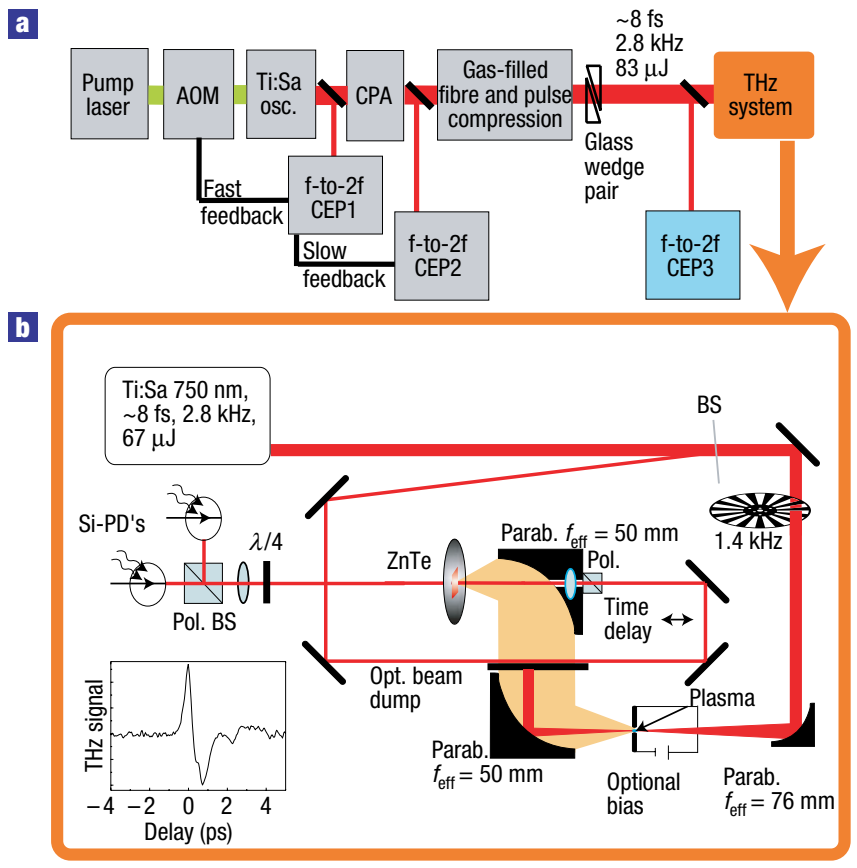

Figure 4 Experimental setup. a, Schematic diagram of the laser system. AOM, acousto-optical modulator; CEP1, f-to-2f interferometer used for CE phase stabilization with fast feedback; CPA, chirped pulse amplification and compression; CEP2, f-to-2f interferometer used for compensation of slow drifts of the CE phase; gas-filled fibre used for spectral broadening; CEP3, f-to-2f interferometer only used for CE phase measurements; BS, beam splitter. b. THz system used for electro-optical THz detection. Inset: A temporal THz wave-form obtained with applied d.c. bias.

incoherent scattering of a majority of the electrons by neighbouring molecules/ions on a timescale of $t_{\mathrm{sc}} \approx 1 \mathrm{fs}$ (see the Methods section). Correspondingly, electrons generated at negative polarity are displaced in the negative direction. Such a collective charge displacement after the passage of the pulse $\left(\lim _{t \rightarrow \infty}\left(\rho^{+}(t)-\right.\right.$ $\left.\rho^{-}(t)\right) \neq 0$ ) creates a quasi-d.c. macroscopic dipole moment. Because of its transient character, it acts as the source of an electromagnetic pulse, whose polarity and field amplitude are determined by the ratio $R_{\rho}^{\infty}=\lim _{t \rightarrow \infty}\left(\left(\rho^{+}-\rho^{-}\right) /\left(\rho^{+}+\rho^{-}\right)\right)$, a quantity that is proportional to the maximal generated dipole moment. A value of $R_{\rho}^{\infty}= \pm 1$ corresponds to the highest possible $\mathrm{THz}$ pulse amplitude, with the polarity of the signal depending on the sign of $R_{\rho}^{\infty}$. For $R_{\rho}^{\infty}=0$, the $\mathrm{THz}$ emission vanishes. Figure 3 displays $R_{\rho}^{\infty}$ as a function of $\varphi_{\mathrm{CE}}$ for laser pulses with gaussian envelopes of various durations $\tau$. It is found that the magnitude of $R_{\rho}^{\infty}$ is directly related to the CE phase with a nearly cosine-like dependence. The contrast, however, rapidly decays when the pulse duration rises towards $10 \mathrm{fs}$.

Although the theory for stereo ATI requires modelling of both the quiver motion and re-collision of electrons, this is not necessary for $\mathrm{THz}$ emission from a plasma generated at atmospheric pressure. The reason for this is that the coherent motion of the majority of electrons is terminated by ultrafast scattering before re-collision, and the $\mathrm{THz}$ signal is proportional to the asymmetry in the tunnelling process. Therefore the signal in our method, for $t_{\mathrm{sc}} \approx 1 \mathrm{fs}$, is directly related to the $\mathrm{CE}$ phase such that a cosine-like pulse $\left(\varphi_{\mathrm{CE}}=0\right)$ will produce a maximum asymmetry in ionization rates and also a maximum $\mathrm{THz}$ signal. A larger $t_{\mathrm{sc}}$ would introduce a phase shift. Finally, the $\mathrm{THz}$ method introduced would directly

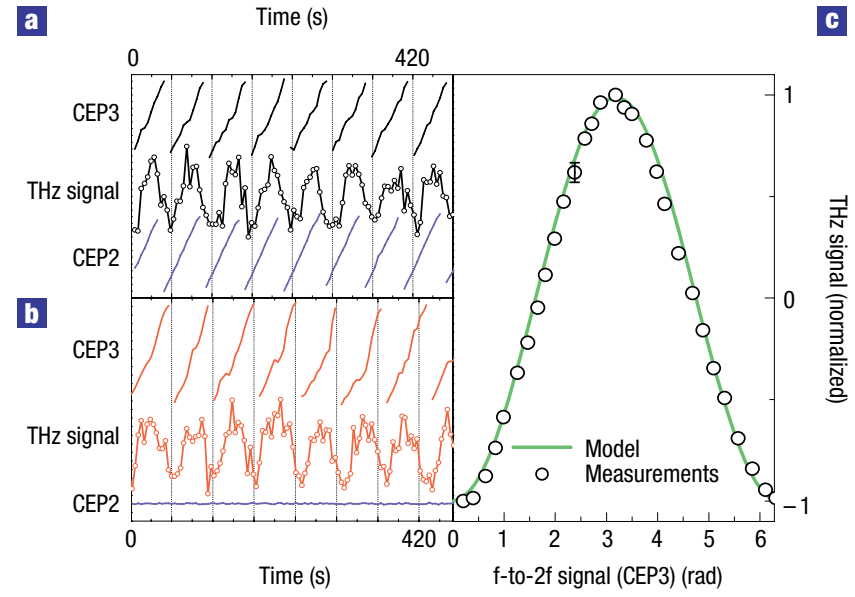

Figure 5 Experimental results. a, Variation of the CE phase of 8-fs pulses obtained by slowly ramping the set-point of the control loop. The CE phase is measured by a f-to-2f interferometer (CEP2) directly behind the laser amplifier, another f-to-2f interferometer (CEP3) behind the recompressor, and by the $\mathrm{THz}$ measurement system by means of electro-optical detection of the THz radiation generated in an ambient air plasma. The integration time constant of the lock-in amplifier in the $\mathrm{THz}$ measurements was $100 \mathrm{~ms}$. In addition, a sliding average was calculated numerically, bringing the effective integration time constant to $5 \mathrm{~s}$. Dispersion effects result in an arbitrary offset between the CE phases measured by CEP2, CEP3 and the THz system. $\mathbf{b}$, Variation of the CE phase of 8-fs pulses by a change of the dispersion in the beam path, realized by two variable glass wedges. c, Adjacent average of the correlation of the CEP3 and the $\mathrm{THz}$ data. The window chosen for averaging was $\pm 0.5 \mathrm{rad}$ of CEP3. For the averaging, the total data pool recorded within 74 min is taken into account. The error bar shows the standard deviation of this averaging. The solid line shows the results of model calculations of $R_{\rho}^{\infty}$ for an 8-fs pulse (constant scaling factor between theory and experiment). The peak electric field used for calculations is $E_{\mathrm{opt}}^{0}=4 \times 10^{10} \mathrm{~V} \mathrm{~m}^{-1}$ corresponding to the expected value in the experiment.

measure the absolute CE phase, if the plasma were a point object ${ }^{27}$. However, the CE phase itself may vary due to dispersion in the plasma, resulting in a concomitant interference of the $\mathrm{THz}$ signals generated along the length of the plasma.

An experimental test of these theoretical findings was carried out by THz-emission spectroscopy with phase-stabilized 8-fs amplified laser pulses (see the Methods section for a detailed description of the setup shown in Fig. 4). We measured the $\mathrm{THz}$ field value from a laser-generated air plasma at a fixed temporal position (that with the largest amplitude of the $\mathrm{THz}$ transients) while continuously varying the CE phase by slowly ramping the set-point of the control loop (with a slope of $2 \pi$ per minute). An exemplary time series spanning $8 \mathrm{~min}$ is shown in Fig. 5a. The $\mathrm{CE}$ phase data measured in the frequency domain by two f-to- $2 \mathrm{f}$ interferometers $^{2}$ (CEP2 and CEP3) are compared with the $\mathrm{THz}$ data. The THz signal varies with the ramped CE phase, as expected from the model calculations shown in Fig. 3.

In a second experiment, the CE phase was kept constant in the oscillator and laser amplifier (CEP2), but varied with the help of a piezo-driven glass-wedge pair behind the pulse compressor and in front of both the third f-to-2f interferometer (CEP3) and the $\mathrm{THz}$ system (also with a phase ramping of $2 \pi$ per minute). The results are shown in Fig. 5b. The measurements again consolidate the expected change of the $\mathrm{THz}$ signal with varying $\mathrm{CE}$ phase.

To provide a more quantitative analysis of the measurements, in Fig. $5 \mathrm{c}$ we show the THz signal as a function of the CEP3 data (open 
circles), averaged over a 74-min time series, where we ramped the phase of the oscillator as in Fig. 5a. The error bar indicates the standard deviation of the $\mathrm{THz}$ signal values. The correlation between $\mathrm{THz}$ signal and CEP3 compares well to the dependence predicted in Fig. 3 for 8 -fs pulses (full line in Fig. $5 \mathrm{c}$ ). The slight deviation of the theoretical data from the experimental data may be a consequence of a non-gaussian shape of the envelope.

The status of the THz approach for CE phase determination can be considered as a proof-of-principle. Nevertheless, to classify its current precision, we compare it with stereo ATI, which achieves a $3 \sigma$ error of $100 \mathrm{mrad}$ for $10 \mathrm{~s}$ of data acquisition and for $6.5-\mathrm{fs} 20-\mu \mathrm{J}$ pulses $^{24}$. The precision reached with the $\mathrm{THz}$ system is $700 \mathrm{mrad}$ for $10 \mathrm{~s}$ of data acquisition for 8 - $\mathrm{fs} 66-\mu \mathrm{J}$ pulses. Considering the factor-of-four improvement to be achieved with 6.5-fs instead of 8 -fs pulses (see Fig. 3), the $\mathrm{THz}$ approach compares well to the much more mature ATI method already. The sensitivity of low-repetition-rate $\mathrm{THz}$-detection can be enhanced significantly by box-car techniques or by $\mathrm{THz}$ interferometry with intensity detection over a large $\mathrm{THz}$-photon bandwidth.

\section{METHODS}

\section{IONIZATION MODEL USED IN THE SIMULATIONS}

We base the quantitative description of the photo-ionization process of atoms and molecules on static-tunnelling theor ${ }^{26}$, with the following expression for the ionization rate:

$$
w_{\text {tun }}=4 \omega_{\mathrm{a}}\left(\frac{U_{\mathrm{ion}}^{\mathrm{N}_{2}}}{U_{\mathrm{ion}}^{\mathrm{H}}}\right)^{5 / 2}\left(\frac{E_{\mathrm{a}}}{E_{\mathrm{opt}}}\right) \exp \left(-\frac{2}{3}\left(\frac{U_{\mathrm{ion}}^{\mathrm{N}_{2}}}{U_{\mathrm{ion}}^{\mathrm{H}}}\right)^{3 / 2}\left(\frac{E_{\mathrm{a}}}{E_{\mathrm{opt}}}\right)\right),
$$

where $\omega_{\mathrm{a}}=4.13 \times 10^{16} \mathrm{~s}^{-1}$ is the atomic frequency unit,

$E_{\mathrm{a}}=5.145 \times 10^{11} \mathrm{~V} \mathrm{~m}^{-1}$ is the atomic unit of the electric field, and $E_{\text {opt }}$ is the optical field strength. $U_{\text {ion }}^{\mathrm{H}}=13.6 \mathrm{eV}$ and $U_{\text {ion }}^{\mathrm{N}_{2}}=15.6 \mathrm{eV}$ denote the ionization potentials of atomic hydrogen and molecular nitrogen, respectively. The simple tunnelling model is known to describe the experimental data for $\mathrm{N}_{2}$ well if the intensity of the laser pulses is above $10^{14} \mathrm{~W} \mathrm{~cm}^{-2}$, and to achieve a fair agreement down to $5 \times 10^{13} \mathrm{~W} \mathrm{~cm}^{-2}$ (ref. 28).

\section{TEMPORAL EVOLUTION OF PLASMA DENSITY AND THZ POLARIZATION}

The emitted $\mathrm{THz}$ field is proportional to the second time derivative of the slowly varying component of the plasma polarization. In the following treatment, we assume that each ionization event contributes the same average dipole moment to this polarization, with the sign depending on the direction of the laser field during each optical half cycle. This reduces the problem to finding the generated plasma densities at each laser field polarity $\rho^{ \pm}$(see below). This approximate model is supported by the following considerations. The spatial amplitude of electron motion for the electric fields considered here is comparable to the average distance between the molecules at atmospheric pressure $(\sim 3 \mathrm{~nm})$. Hence, it is probable that a large fraction of the electrons collide with neighbouring atoms and ions during the first excursion away from their parent ions. This scattering will terminate their coherent contribution to the plasma polarization on the associated timescale $t_{\mathrm{sc}} \approx 1 \mathrm{fs}$. We find experimental evidence for such ultrafast scattering in measurements of the type reported in ref. 8 but extended to above-ambient pressure (data to be published). It is observed that the $\mathrm{THz}$ signal strongly decreases above $\sim 2 \mathrm{bar}$, which can be explained by a further decrease in the scattering time below that of an optical half cycle.

We compute the plasma densities $\rho^{+}(t)$ and $\rho^{-}(t)$ by solving the following coupled differential equations:

$$
\begin{aligned}
& \frac{\mathrm{d}}{\mathrm{d} t} \rho^{+}(t)= \begin{cases}w_{\text {tun }}\left(E_{\mathrm{opt}}(t)\right) \cdot\left(\rho_{\mathrm{gas}}^{0}-\rho^{+}(t)-\rho^{-}(t)\right), & \text { if } E_{\mathrm{opt}}(t) \geq 0 \\
0, & \text { if } E_{\mathrm{opt}}(t)<0,\end{cases} \\
& \frac{\mathrm{d}}{\mathrm{d} t} \rho^{-}(t)= \begin{cases}w_{\mathrm{tun}}\left(E_{\mathrm{opt}}(t)\right) \cdot\left(\rho_{\mathrm{gas}}^{0}-\rho^{+}(t)-\rho^{-}(t)\right), & \text { if } E_{\mathrm{opt}}(t)<0 \\
0, & \text { if } E_{\mathrm{opt}}(t) \geq 0 .\end{cases}
\end{aligned}
$$

Here, $\rho_{\text {gas }}^{0}=2.7 \times 10^{19} \mathrm{~cm}^{-3}$ is the density of $\mathrm{N}_{2}$ molecules at atmospheric pressure assuming that air only consists of $\mathrm{N}_{2}$. $E_{\text {opt }}(t)$ denotes the electric field strength of the laser pulse. We neglect plasma recombination, which occurs on a much longer timescale ${ }^{29}$. We assume the following temporal wave-form of the laser pulse:

$$
E_{\mathrm{opt}}(t)=E_{\mathrm{opt}}^{0} A(t) \cos \left(\frac{2 \pi c}{\lambda} t+\varphi_{\mathrm{CE}}\right)
$$

where $A(t)$ is a gaussian envelope. Although such a product separation of carrier and envelope can become ill-defined as the pulse duration approaches the sub-cycle range ${ }^{30}$, it is perfectly reasonable for the shortest pulse durations we consider here. $E_{\mathrm{opt}}^{0}$ is assumed to be limited in the calculations as a consequence of self-defocusing of the light beam in the plasma ${ }^{31}$. The $E_{\text {opt }}^{0}$ value in the calculations in this paper is chosen so that the resulting total plasma density is $10 \%$.

\section{LIGHT SOURCE}

The experiments were carried out with a light source as shown in Fig. 4a. A commercially available Ti:sapphire Multipass CPA System 'Femtopower Compact Pro' (Femtolasers) is seeded with a self-built laser oscillator (pulse energy $4 \mathrm{~nJ}$, pulse duration $20 \mathrm{fs}$, centre wavelength $810 \mathrm{~nm}$, CE phase stabilized with $\mathrm{f}$-to- $2 \mathrm{f}$ self-referencing ${ }^{1}(\mathrm{CEP} 1)$ ) and driven at a repetition rate of $2.8 \mathrm{kHz}$. Its output ( $500 \mu \mathrm{J}, 30 \mathrm{fs}$, at $805 \mathrm{~nm}$ ) is focused into a gas-filled hollow fibre (inner diameter $200 \mu \mathrm{m}$, length $82 \mathrm{~cm}, 4.5$ bar neon). A small fraction of the CPA output (few $\mu \mathrm{J}$ ) are used for a f-to-2f interferometer ${ }^{2}$ (CEP2) to correct for slow CE phase drifts in the amplifier. The fibre output is then compressed to its bandwidth-limited duration of $8 \mathrm{fs}$ using dispersive mirrors and a prism-compressor sequence with two fused silica prisms, with final output parameters $(83 \mu \mathrm{J}, 8 \mathrm{fs}$, at $750 \mathrm{~nm}$ (centre wavelength)).

\section{THZ-EMISSION SPECTROSCOPY SYSTEM}

$80 \%$ of the final output is fed into the THz detection setup, which is shown in Fig. 4 b. $10 \%$ (the residual $10 \%$ are unused) is used for a third f-to- $2 \mathrm{f}$ interferometer ${ }^{2}$ (CEP3), which traces CE phase changes for direct comparison with the $\mathrm{THz}$ data in respect to a constant arbitrary offset. The $\mathrm{THz}$ system is a typical $\mathrm{THz}$ pump-probe measurement system with balanced electro-optical $\mathrm{THz}$ detection ${ }^{5,8}$, the whole setup is fitted on a $30 \times 45-\mathrm{cm}$ breadboard. The pump beam is mechanically chopped at half the laser repetition rate. The plasma is generated in the focus of an off-axis paraboloidal mirror with an effective focal length of $76 \mathrm{~mm}$. For optimization of the alignment and a first determination of the zero-delay point for the $\mathrm{THz}$ detection, an additional d.c. bias field can be applied to the focal region ${ }^{5}$. The probe beam passes along a variable delay line and is focused collinearly with the THz beam into the electro-optical ZnTe detector crystal. The $\mathrm{THz}$ wave-form, as measured with a d.c.-biased air plasma, is shown in the lower left part of Fig. 4b. For the CE phase measurements, the delay stage of the probe beam is positioned at the zero-delay point where the strongest $\mathrm{THz}$ signal is obtained, and the external bias field is switched off.

\section{Received 16 December 2005; accepted 24 March 2006; published 23 April 2006.}

\section{References}

1. Baltuška, A. et al. Attosecond control of electronic processes by intense light fields. Nature $\mathbf{4 2 1}$ 611-615 (2003)

2. Baltuška, A. et al. Phase-controlled amplification of few-cycle laser pulses. IEEE J. Sel. Top. Quantum Electron. 9, 972-989 (2003).

3. Apolonski, A. et al. Observation of light-phase-sensitive photoemission from a metal. Phys. Rev. Lett. 92, 073902 (2004).

4. Paulus, G. G. et al. Absolute-phase phenomena in photoionization with few-cycle laser pulses. Nature 414, 182-184 (2001).

5. Löffler, T., Jacob, F. \& Roskos, H. G. Generation of terahertz pulses by photoionization of electrically biased air. Appl. Phys. Lett. 77, 453-455 (2000).

6. Löffler, T. \& Roskos, H. G. Gas-pressure dependence of terahertz-pulse generation in a laser-generated nitrogen plasma. J. Appl. Phys. 91, 2611-2614 (2002).

7. Cook, D. J. \& Hochstrasser, R. M. Intense terahertz pulses by four-wave rectification in air. Opt. Lett. 25, 1210-1212 (2000).

8. Kreß, M., Löfler, T., Eden, S., Thomson, M. \& Roskos, H. G. Terahertz-pulse generation by photoionization of air with laser pulses composed of both fundamental and second-harmonic wave. Opt. Lett. 29, 1120-1122 (2004).

9. Bartel, T., Gaal, P., Reimann, K., Woerner, M. \& Elsaesser, T. Generation of single-cycle THz transients with high electric-field amplitudes. Opt. Lett. 30, 2805-2807 (2005).

10. Xie, X., Dai, J. \& Zhang, X.-C. Coherent control of THz wave generation in ambiant air. Phys. Rev. Lett. 96, 075005 (2006).

11. Itatani, J. et al. Controlling high harmonic generation with molecular wave packets. Phys. Rev. Lett. 94, 123902 (2005).

12. Macklin, J. J., Kmetec, J. D. \& Gordon III, C. L. High-order harmonic generation using intense femtosecond pulses. Phys. Rev. Lett. 70, 766-769 (1993).

13. Gordienko, S., Pukhov, A., Shorokhov, O. \& Beava, T. Relativistic Doppler effect: Universal spectra and zeptosecond pulses. Phys. Rev. Lett. 93, 115002 (2004). 
14. Itatani, J. et al. Tomographic imaging of molecular orbitals. Nature 432, 867-871 (2004).

15. Pukhov, A. \& Meyer-ter-Vehn, J. Laser wake field acceleration: The high non-linear broken-wave regime. Appl. Phys. B 74, 355-361 (2002).

16. Mangles, S. P. D. et al. Monoenergetic beams of relativistic electrons from intense laser-plasma interactions. Nature 431, 535-538 (2004).

17. Geddes, C. G. R. et al. High-quality electron beams from a laser wakefield accelerator using plasma-channel guiding. Nature 431, 538-541 (2004).

18. Faure, J. et al. A laser-plasma accelerator producing monoenergetic electron beams. Nature 431, 541 (2004)

19. Liu, X. et al. Nonsequential double ionization at the single-optical-cycle limit. Phys. Rev. Lett. 93, 263001 (2004).

20. Lindner, F. et al. Gouy-phase shift for few-cycle laser pulses. Phys. Rev. Lett. 92, 113001 (2004).

21. Roos, P. A. et al. Solid-state carrier-envelope phase stabilization via quantum interference control of injected photocurrents. Opt. Lett. 30, 735-737 (2005).

22. Bandrauk, A. D., Chelkowski, S. \& Nguyen, H. S. Attosecond localization in molecules. Int. J. Quantum Chem. 100, 834-844 (2004).

23. Paulus, G. G. et al. Measurement of the phase of few-cycle laser pulses. Phys. Rev. Lett. 91, 253004 (2003)

24. Paulus, G. G. A meter of the "absolute" phase of few-cycle pulses. Laser Phys. 15, 843-854 (2005).

25. Hamster, H., Sullivan, A., Gordon, S., White, W. \& Falcone, R. W. Subpicosecond electromagnetic pulses from intense laser-plasma interaction. Phys. Rev. Lett. 71, 2725-2728 (1993).

26. Corkum, P. B., Burnett, N. H. \& Brunel, F. Above-threshold ionization in the long-wavelength limit. Phys. Rev. Lett. 62, 1259-1262 (1989).
27. Löffler, T., Kreß, M., Thomson, M. \& Roskos, H. G. Efficient terahertz pulse generation in laser-induced gas plasmas. Act. Phys. Pol. A 107, 99-108 (2005)

28. Guo, C., Li, M., Nibarger, J. P. \& Gibson, G. N. Single and double ionization of diatomic molecules in strong laser fields. Phys. Rev. A 58, 4271-4274 (1998).

29. Biondi, M. A. \& Brown, S. C. Measurement of electron-ion recombination. Phys. Rev. 76, 1697-1700 (1949).

30. Shvartsburg, A. B. in Time-Domain Optics of Ultrashort Waveforms (Oxford Series in Optical Sciences, Vol. 10, Oxford Univ. Press, Oxford, 1996).

31. Mlejnek, M., Wright, E. M. \& Moloney, J. V. Femtosecond pulse propagation in argon: A pressure dependence study. Phys. Rev. E 58, 4903-4910 (1998).

\section{Acknowledgements}

We would like to acknowledge fruitful discussions with M. Horbatsch from York University, Canada. This work was supported in part by the Hochschulförderungsprogramm of GSI Darmstadt and by the Deutsche Forschungsgemeinschaft within contract Mo850/2 (U.M.). Support from the Leibniz-Program of the Deutsche Forschungsgemeinschaft (J.U.) is gratefully acknowledged. Correspondence and requests for materials should be addressed to M.K.

Competing financial interests

The authors declare that they have no competing financial interests.

Reprints and permission information is available online at http://npg.nature.com/reprintsandpermissions/ 